\title{
Evidence for magnetic fractional excitations in a Kitaev quantum-spin-liquid candidate $\alpha-\mathrm{RuCl}_{3}$
}

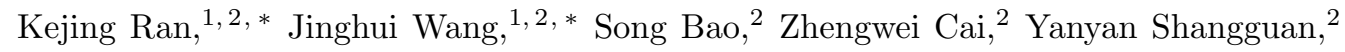 \\ Zhen Ma, ${ }^{2,}{ }^{3}$ Wei Wang, ${ }^{4}$ Zhao-Yang Dong, ${ }^{5}$ P. Čermák,,${ }^{6,7}$ A. Schneidewind, ${ }^{6}$

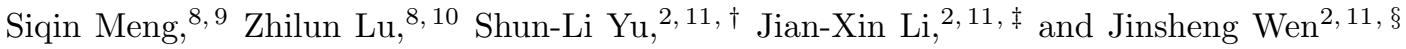 \\ ${ }^{1}$ School of Physical Science and Technology and ShanghaiTech Laboratory for Topological Physics, \\ ShanghaiTech University, Shanghai 200031, China \\ ${ }^{2}$ National Laboratory of Solid State Microstructures and Department of Physics, Nanjing University, Nanjing 210093, China \\ ${ }^{3}$ Institute for Advanced Materials, Hubei Normal University, Huangshi 435002, China \\ ${ }^{4}$ School of Science, Nanjing University of Posts and Telecommunications, Nanjing 210023, China \\ ${ }^{5}$ Department of Applied Physics, Nanjing University of Science and Technology, Nanjing 210094, China \\ ${ }^{6}$ Jülich Centre for Neutron Science (JCNS) at Heinz Maier-Leibnitz Zentrum (MLZ), \\ Forschungszentrum Jülich GmbH, Lichtenbergstr. 1, 85748 Garching, Germany \\ ${ }^{7}$ Charles University, Faculty of Mathematics and Physics, \\ Department of Condensed Matter Physics, Ke Karlovu 5, 121 16, Praha, Czech Republic \\ ${ }^{8}$ Helmholtz-Zentrum Berlin für Materialien und Energie GmbH, Hahn-Meitner-Platz 1D-14109 Berlin, Germany \\ ${ }^{9}$ China Institute of Atomic Energy, Beijing 102413, China \\ ${ }^{10}$ The Henry Royce Institute and Department of Materials Science and Engineering, \\ The University of Sheffield, Sir Robert Hadfield Building, Sheffield, S1 3JD, United Kingdom \\ ${ }^{11}$ Collaborative Innovation Center of Advanced Microstructures, Nanjing University, Nanjing 210093, China
}

\begin{abstract}
$\alpha-\mathrm{RuCl}_{3}$ has been studied extensively because of its proximity to the Kitaev quantum-spin-liquid (QSL) phase and the possibility of approaching it by tuning the competing interactions. Here we present the first polarized inelastic neutron scattering study on $\alpha-\mathrm{RuCl}_{3}$ single crystals to explore the scattering continuum around the $\Gamma$ point at the Brillouin zone center, which was hypothesized to be resulting from the Kitaev QSL state but without concrete evidence. With polarization analyses, we find that while the spin-wave excitations around the $M$ point vanish above the transition temperature $T_{\mathrm{N}}$, the pure magnetic continuous excitations around the $\Gamma$ point are robust against temperature. Furthermore, by calculating the dynamical spin-spin correlation function using the cluster perturbation theory, we derive magnetic dispersion spectra based on the $K-\Gamma$ model, which involves with a ferromagnetic Kitaev interaction of $-7.2 \mathrm{meV}$ and an off-diagonal interaction of $5.6 \mathrm{meV}$. We find this model can reproduce not only the spin-wave excitation spectra around the $\mathrm{M}$ point, but also the non-spin-wave continuous magnetic excitations around the $\Gamma$ point. These results provide evidence for the existence of fractional excitations around the $\Gamma$ point originating from the Kitaev QSL state, and further support the validity of the $K-\Gamma$ model as the effective minimal spin model to describe $\alpha-\mathrm{RuCl}_{3}$.
\end{abstract}

Quantum spin liquids (QSLs) with fractionalized spin excitations and long-range entanglement have drawn a lot of attention since Anderson first proposed the QSL state within the resonating-valence-bond (RVB) model [1] and used it to explain the high-temperature superconductivity [2. Typically, QSL states have been proposed in triangular and kagome lattices where antiferromagnetic interactions are highly frustrated due to the geometrical constrain, and therefore there remain strong quantum fluctuations in these systems that prevent spins from establishing a long-range order as observed in a usual magnet at low temperatures 3 -7. Besides the RVB model which was built upon geometrical frustration, the Kitaev model defined on the ideal two-dimensional honeycomb lattice with spin $S=1 / 2$, is an exactly solvable model with the QSL ground state $[8$. On a honeycomb lattice, there is no geometrical frustration, and it is the anisotropic bond-dependent Kitaev interactions that lead to the frustration on a single site and give rise to the "Kitaev" QSL state 8, 9. A Kitaev QSL can host fractional excitations represented by e.g., Majo- rana fermions, which behave as anyons obeying the nonAbelien statistics under magnetic field, and allow to be braided for fault-tolerant topological quantum computation [8, 10 12].

In general, for a spin-only system, it is unrealistic to find the bond-dependent Kitaev interactions which underlie the Kitaev QSL, as the spin interactions ought to be isotropic along the three symmetry-equivalent bonds in a honeycomb lattice. One plausible approach of realizing the anisotropic Kitaev interaction is to resort to spin-orbital-coupling (SOC) assisted Mott insulators, where the effective moment $J_{\text {eff }}=1 / 2$ is an entanglement of the orbital moment $L=1$ and spin moment $S=1 / 2$ [9, 13, 14]. This idea was initially applied to iridates such as $\mathrm{Na}_{2} \mathrm{IrO}_{3}$ where iridium and oxygen ions form edge-shared $\mathrm{IrO}_{6}$ octahedra, and the $5 d$ iridium ions with strong SOC and $J_{\text {eff }}=1 / 2$ form a two-dimensional honeycomb network [13, 15, 22, The geometrical configuration suppresses the isotropic Heisenberg interaction while on the other hand promotes the anisotropic Kitaev interaction in the aid of the large SOC and strong 
spatial anisotropy of the $d$ orbitals [13, 23]. These features should have made iridates a promising platform to investigate the Kitaev physics. However, these materials suffer from the following problems: i) the presence of monoclinic and trigonal distortions renders the applicability of the localized $J_{\text {eff }}$ picture to these materials questionable 22, 24, 25; ii) iridium has a large neutron absorption cross section and thus it is difficult to carry out inelastic neutron scattering (INS) measurements to extract the magnetic interactions; iii) large-size and highquality single crystals are not available [22].

Therefore, more efforts have been devoted into $\alpha$ $\mathrm{RuCl}_{3}$ with the honeycomb lattice recently, which can also realize the $\mathrm{SOC}$-assisted $J_{\text {eff }}=1 / 2$ Mott insulating state, despite the weaker SOC effect of the $4 d$ $\mathrm{Ru}^{3+}$ [26, 27]. Although the low-temperature phase is not the long-sought Kitaev QSL but zigzag ordered phase instead 27 31, it is shown to exhibit salient Kitaev interactions due to the close-to-ideal bond configuration in the $\mathrm{RuCl}_{6}$ octohedron [29, 31, 39. Notably, the zigzag order is close to the Kitaev QSL phase in the phase diagram and is fragile subject to external magnetic field [28, 4046] or pressure [47 49]. More intriguingly, a moderate in-plane magnetic field may drive the system into a QSL phase [50 53]. In the zigzag phase, INS experiments show that the excitations are mostly concentrated around the $\mathrm{M}$ and $\Gamma$ points of the two-dimensional Brillouin zone, as sketched in Fig. 1(a). While it is generally believed the gapped sharp excitations around the $\mathrm{M}$ point are spin waves associated with the magnetic order, the origin of the broad continuum around the $\Gamma$ point is still controversial 29, 31, 37, 54, 60. On the one hand, various spectroscopic results, such as Raman scattering [55, 56, optical $\mathrm{THz}$ spectroscopy [57,59, and INS spectra [29, 37, 60], show a nearly temperature-independent excitation continuum around the $\Gamma$ point, which is reminiscent of the fractionalized excitations originating from the Kitaev QSL state. On the other hand, because of the small scattering angle around the $\Gamma$ point, the broad continuous feature in the INS experiment could be due to the nuclear scattering 37, which is also temperature independent. In addition, it has been argued that the continuum feature may originate from the incoherent excitations caused by strong magnetic anharmonicity 54 . Therefore, it is critical to distinguish whether the continuum is fractional magnetic excitation or nuclear scattering. Polarized neutron scattering experiment from which pure magnetic scattering can be obtained will help solve this problem 61, 62. However, because of the much reduced scattering cross section in a polarized neutron experiment, it is very challenging to obtain meaningful results for thin single crystals like $\alpha-\mathrm{RuCl}_{3}$.

In this Letter, we report polarized neutron scattering study on 2 -g high-quality $\alpha-\mathrm{RuCl}_{3}$ single crystals. The data allow us to distinguish magnetic scattering from nuclear scattering. Our polarization results show that the
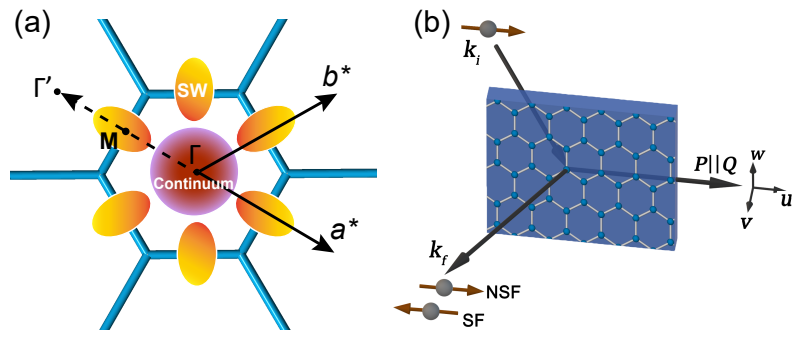

FIG. 1. (a) Schematic of the magnetic excitations in the first Brillouin zone for $\alpha-\mathrm{RuCl}_{3}$ in the zigzag magnetic order state. Thick solid lines denote the Brillouin zone boundary. Excitations around the $\Gamma$ point represent the scattering continuum. The six-fold symmetric excitations around the M point denote spin-wave excitations. (b) Schematic of the experimental setup for the polarized neutron scattering measurements for the case of $\boldsymbol{P} \| \boldsymbol{Q} . \boldsymbol{P}$ is the neutron polarization direction, and $\boldsymbol{Q}$ is the scattering vector, obtained by $\boldsymbol{Q}=\boldsymbol{k}_{\mathrm{i}}-\boldsymbol{k}_{\mathrm{f}} . \boldsymbol{k}_{\mathrm{i}}$ and $\boldsymbol{k}_{\mathrm{f}}$ are the initial and final wave vector of neutrons, respectively. Neutron polarization directions parallel, up and perpendicular, with respect to $\boldsymbol{Q}$ are labeled by $\boldsymbol{u}, \boldsymbol{w}$ and $\boldsymbol{v}$, respectively. SF and NSF denote spin-flip and non-spin-flip of neutrons, respectively.

broad continuous excitations around the $\Gamma$ point are of magnetic origin, which are persistent at temperatures far above the magnetic ordering temperature $T_{\mathrm{N}}$. In contrast, the spin-wave excitations around the $\mathrm{M}$ point disappear above $T_{\mathrm{N}}$. Moreover, by comparing the calculation results using Kitaev-off-diagonal $(K-\Gamma)$ and KitaevHeisenberg $(K-J)$ models with the unpolarized neutron experimental data, we find that the $K-\Gamma$ model with a ferromegntic $K=-7.2 \mathrm{meV}$ and $\Gamma=5.6 \mathrm{meV}$ can describe both the low-energy response of the spin-wave excitations associated with the zigzag ordered state around the M point, and the continuous magnetic excitations around the $\Gamma$ point. These results are consistent with the presence of fractional magnetic excitations in $\alpha-\mathrm{RuCl}_{3}$ and further support the $K-\Gamma$ model to be the minimal effective spin model in describing the system.

High-quality single crystals of $\alpha-\mathrm{RuCl}_{3}$ were grown using the chemical-vapor-transport method with $\alpha-\mathrm{RuCl}_{3}$ powders. The sheet-like crystals had a natural $a-b$ plane as the cleavage plane and had a typical size of $10 \times 10 \times 1 \mathrm{~mm}^{3}$, which weighed about $60 \mathrm{mg}$ a piece. Both susceptibility measurements and specific heat measurements showed a sharp antiferromagnetic phase transition at $\sim 7.5 \mathrm{~K}$ [31, 44, 51]. Polarized neutron scattering measurements were performed on PANDA, a cold neutron triple-axis spectrometer with the polarized option located at FRM II 63. $X Y Z$-difference method for neutron polarization analysis 64 was used to separate the magnetic scattering from nuclear coherent, nuclear spin incoherent, isotopic incoherent and background contributions. Unpolarized neutron scattering measurements were carried out on a cold neutron triple-axis spec- 

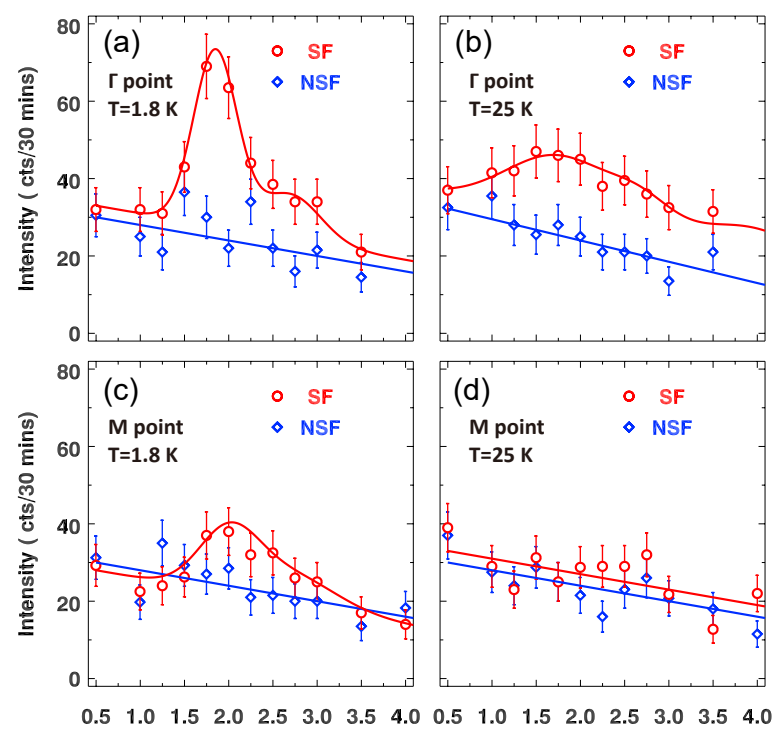

$E(\mathrm{meV})$

$E(\mathrm{meV})$

FIG. 2. (a) and (b) Energy scans of $\alpha-\mathrm{RuCl}_{3}$ at the $\Gamma$ point $(0,0,1.5)$ measured at 1.8 and $25 \mathrm{~K}$ with polarized neutrons in the spin-flip (SF) and non-spin-flip (NSF) modes. The neutron polarization used was $\boldsymbol{P} \| \boldsymbol{Q}$. (c) and (d) Same scans as in (a) and (b) but at the M point $(0.5,0,0)$. Lines through data are guides to the eye. Errors represent one standard deviation throughout the paper.

trometer FLEXX located at HZB. We used a fixed final energy mode with $E_{\mathrm{f}}=5 \mathrm{meV}$, and a double-focusing condition for both the monochromator and analyzer for all these experiments. For the experiment on PANDA, about 45 single crystals weighed about $2 \mathrm{~g}$ in total were co-aligned together on two aluminum plates using a Laue x-ray diffractometer. Heusler alloys with the $(1,1,1)$ reflection plane were used as the monochromator and analyzer. Guide magnetic fields were used to rotate the neutron polarization direction along $(\boldsymbol{P} \| \boldsymbol{Q})$ or vertical to the scattering wave vector $(\boldsymbol{P} \perp \boldsymbol{Q})$. A Mezei coil flipper was used between the sample and analyzer to flip the polarization of neutrons. The flipping ratios of the three directions $\boldsymbol{u}, \boldsymbol{v}$ and $\boldsymbol{w}$ we labeled in Fig. 1(b) were $18.1,7.5,16.9$, respectively. We denote a scattering event in which the spin makes a $180^{\circ}$ rotation as spin flip (SF), and scattering with no spin direction change as non spin flip (NSF). For the measurements on FLEXX, we used 24 single crystals with a total mass of $\sim 2.2 \mathrm{~g}$. All the experiments were conducted in the $(H O L)$ plane. The sample mosaic was about 3.6 degrees at PANDA and 1.93 degrees at FLEXX scanned around the Bragg peak $(0,0,3)$. We used the $\mathrm{P} 3_{1} 12$ notation with $a=b=5.96 \AA$, and $c=17.2 \AA$ as the lattice constants. The wave vector $\boldsymbol{Q}$ was described by $(H K L)$ reciprocal lattice units (r.l.u.) of $\left(a^{*}, b^{*}, c^{*}\right)=(4 \pi / \sqrt{3} a, 4 \pi / \sqrt{3} b, 2 \pi / c)$.

According to the polarized neutron scattering rules [65], when the neutron polarization direction $\boldsymbol{P}$ is
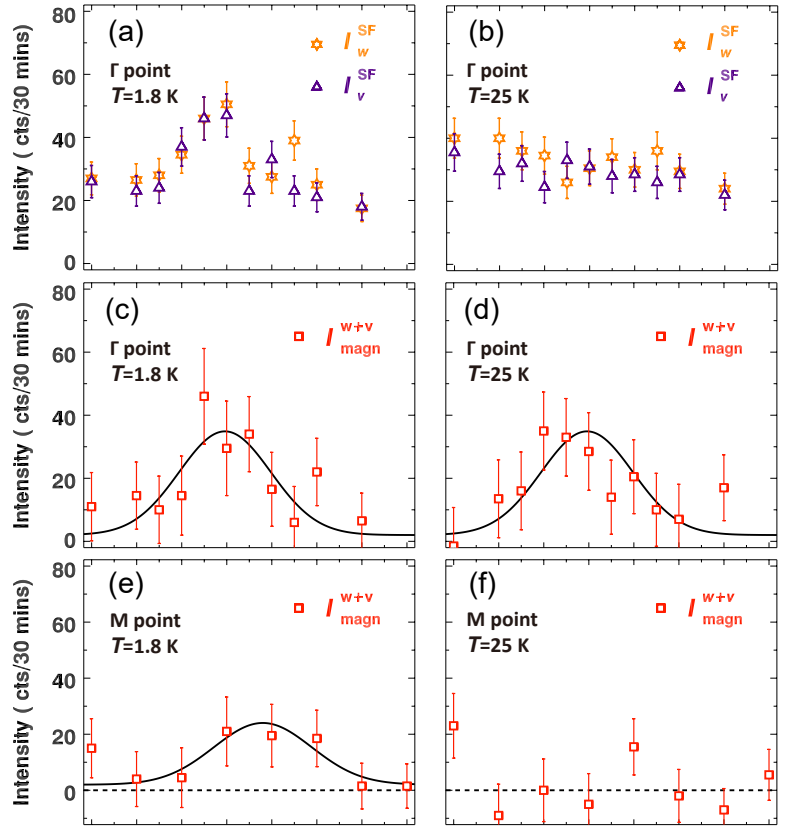

$\begin{array}{llllllllllllllll}0.5 & 1.0 & 1.5 & 2.0 & 2.5 & 3.0 & 3.5 & 4.0 & 0.5 & 1.0 & 1.5 & 2.0 & 2.5 & 3.0 & 3.5 & 4.0\end{array}$

$E$ (meV)

$E$ (meV)

FIG. 3. (a) and (b) Energy scans at the $\Gamma$ point measured at 1.8 and $25 \mathrm{~K}$ with polarized neutrons in the SF mode. The neutron polarization used was $\boldsymbol{P} \perp \boldsymbol{Q} . I_{v}^{\mathrm{SF}}$ and $I_{w}^{\mathrm{SF}}$ are scattering intensities for SF mode in directions of perpendicular and up with respect to $\boldsymbol{Q}$. (c) and (d) Pure magnetic intensities around the $\Gamma$ point measured at 1.8 and $25 \mathrm{~K}$, obtained by $I_{\mathrm{mag}}^{w+v}=I_{\mathrm{mag}}^{w}+I_{\mathrm{mag}}^{v}=2 I_{u}^{\mathrm{SF}}-I_{v}^{\mathrm{SF}}-I_{w}^{\mathrm{SF}}$. (e) and (f) Same as in (c) and (d) but at the M point. Solid lines through data are guides to the eye. Dashed lines denote the background.

parallel to the scattering vector $\boldsymbol{Q}$, magnetic and nuclear scatterings are detected in the SF and NSF channels, respectively. In the experiment, we polarized neutrons along the scattering vector first. Here we take $(0,0,1.5)$ as the $\Gamma$ point since neutron scattering experiment cannot access the $\Gamma$ point at $\boldsymbol{Q}=0$. Figure 2 shows energy scans at the $\Gamma$ and $\mathrm{M}$ points $(0.5,0,0)$ at $1.8 \mathrm{~K}$ (below the $T_{\mathrm{N}}$ of $7.5 \mathrm{~K}$ ) and $25 \mathrm{~K}$ (well above the $T_{\mathrm{N}}$ ). As shown in Fig. 2(a), pronounced magnetic excitations are observed in the SF channel at $T=1.8 \mathrm{~K}$ at the $\Gamma$ point. There is a clear peak centered at about $1.8 \mathrm{meV}$. On the other hand, the data in the NSF channel is essentially a sloping background. These results indicate that the excitations at the $\Gamma$ point detected in the unpolarized INS experiments previously are of magnetic origin $[37,45,60$. When we increase the temperature up to $25 \mathrm{~K}$, in the SF channel, there are still clear magnetic excitations on top of the sloping background, as shown in Fig. 2(b). The magnetic excitations are significantly broader than those in the magnetically ordered state. This broad and continuum-like feature is a characteristic of the fractional excitations [37, 60]. We suspect that the reason why intensities at low temperature are stronger than those at 

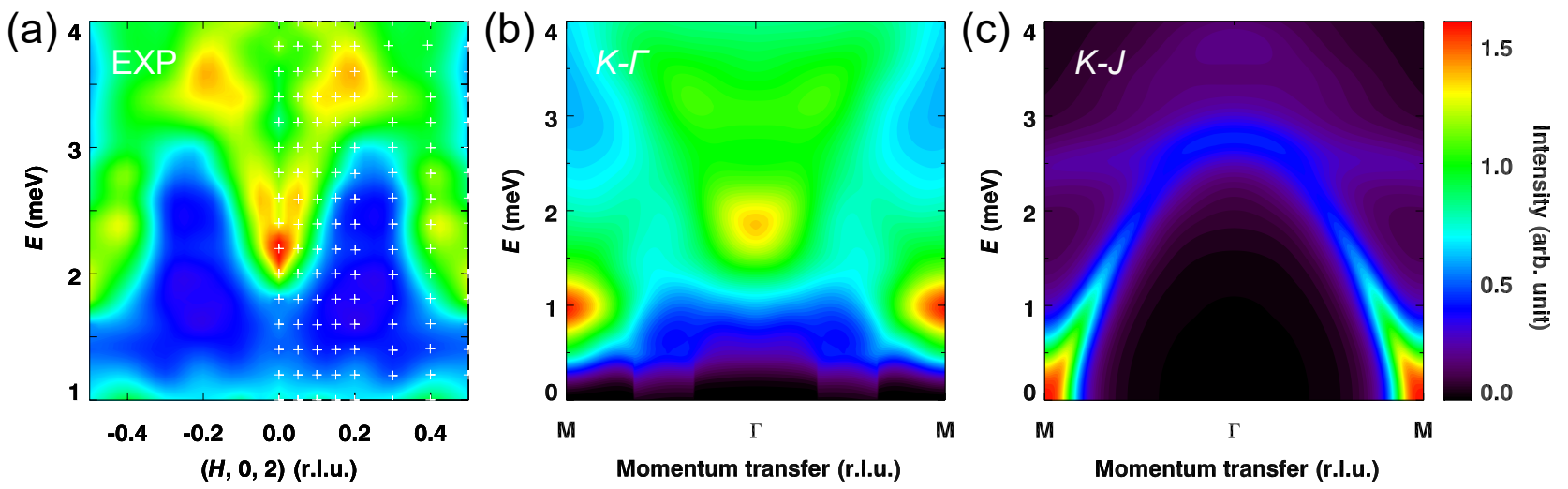

FIG. 4. (a) Magnetic dispersion along [H00] at $\mathrm{T}=1.8 \mathrm{~K}$ obtained by plotting the points marked with white cross dots. Left side of the dispersion from $H=-0.5$ to 0 r.l.u is the mirror symmetrical figure on the right from $H=0$ to 0.5 r.l.u. (b) Magnetic excitation spectrum of the $K-\Gamma$ model for $\Gamma / K=-0.73$ with $K<0$. (c) Theoretical magnetic excitation spectra for the zigzag phases of the $K-J$ model for $J / K=-0.36$ with $K>0$.

high temperature is that the low-temperature excitations have some interactions with the spin-wave excitations. For comparison, we also measured the excitations at the $\mathrm{M}$ point, which is the wave vector of the zigzag magnetic order 27, 31. As shown in Fig. 2(c), a magnetic peak is observed at $T=1.8 \mathrm{~K}$ in the $\mathrm{SF}$ channel on top of the sloping background obtained in the NSF channel. As expected, this peak disappears when the temperature is above the $T_{\mathrm{N}}$, consistent with it being the spin-wave excitations arising from the zigzag magnetic order [29, 31, 37. The different temperature dependence of the excitations at the $\Gamma$ and $\mathrm{M}$ points and the persistence of the magnetic excitations at the $\Gamma$ point further prove that these exciations are magnetic, fractionalized, and robust within a certain temperature window $[60,66$.

Although all the magnetic scattering is present in the SF channel when $\boldsymbol{P} \| \boldsymbol{Q}$, the total intensity contains some background resulting from the incoherent nuclear spin scattering with an intensity contribution of $\frac{2}{3} I_{\text {inc }}^{\text {spin }} 65$. In order to deduct this term and obtain pure magnetic scattering intensities, we polarized the neutron beam to the two directions $\boldsymbol{v}$ and $\boldsymbol{w}$ perpendicular to $\boldsymbol{Q}$ as illustrated in Fig. 1(b) and performed the same scans as in Fig. 2 to obtain $I_{v}^{\mathrm{SF}}$ and $I_{w}^{\mathrm{SF}}$, as shown in Fig. 3(a) and (b). Pure magnetic scattering intensities are given by $I_{\mathrm{mag}}^{w+v}=I_{\mathrm{mag}}^{w}+I_{\mathrm{mag}}^{v}=2 I_{u}^{\mathrm{SF}}-I_{v}^{\mathrm{SF}}-I_{w}^{\mathrm{SF}}$ 65. The soobtained intensities at the $\Gamma$ point are shown in Fig. 3(c) and (d). It can be seen that they exist at both low and high temperatures. The temperature-stable magnetic excitations at the $\Gamma$ point are evident of the fractional excitations which are originated from the Kitaev QSL state. For comparison, the pure magnetic intensities at the M point are shown in Fig. 3(e) and (f). In contrast to those at the $\Gamma$ point, the magnetic excitations at the $M$ point are only observed at the low temperature. The observations that the pure magnetic intensities $I_{\mathrm{mag}}^{w+v}$ at the $\Gamma$ point remain constant against temperature while those of spin waves at the $\mathrm{M}$ point are completely vanishing above the $T_{\mathrm{N}}$ indicate the emergence of fractional excitations at a higher temperature above the $T_{\mathrm{N}}[60,66$.

After pinning down the origin of the excitations at the $\Gamma$ point, we next discuss the effective spin model to describe the magnetic excitations at both the $\Gamma$ and $M$ points, in contrast to previous attempts where the excitations around the two momenta were treated separately using different models [29, 31, 35, 37. To do this, we first performed unpolarized INS measurements at $T=1.8 \mathrm{~K}$ to obtain the magnetic dispersion as shown in Fig. 4(a). There are clear magnetic excitations weakly dispersing from both the $\Gamma$ and $\mathrm{M}$ points. A broad continuum with a gap of $\sim 1.8 \mathrm{meV}$ appears around the $\Gamma$ point, consistent with the polarization data and earlier results [37, 45, 60. To describe these data, we first consider the $K-\Gamma$ model that we used to fit the spin-wave excitations in our previous works [31, 35. Its Hamiltonian can be written as,

$$
H_{K-\Gamma}=\sum_{<i j>}\left[K S_{i}^{\gamma} S_{j}^{\gamma}+\Gamma\left(S_{i}^{\alpha} S_{j}^{\beta}+S_{i}^{\beta} S_{j}^{\alpha}\right)\right] .
$$

Here, $\gamma=x, y, z$ labels the three nearest-neighbor bonds on the honeycomb lattice, and $(\alpha, \beta, \gamma)$ is a permutation of $(x, y, z)$ with $\alpha$ and $\beta$ labeling the two remaining directions on a $\gamma$ bond. We calculated the dynamical spin-spin correlation function using the cluster perturbation theory, which has been successfully applied to other magnetically frustrated systems [67]. The calculated results with one typical parameter set of ferromagnetic $K=-7.2 \mathrm{meV}$ and $\Gamma=5.6 \mathrm{meV}$ are shown in Fig. 4(b). These parameters ensures that the system is in the zigzag order phase [19, 35], and are consistent with our previous works 31,35 . Compared to the experimental spectra shown in Fig. 4(a), it is clear that both the excitations at the $M$ and $\Gamma$ points can be well captured 
by this model. As we demonstrated in Refs. [31, 35, the spin-wave excitations should only be observed to disperse from the $\mathrm{M}$ point around $2 \mathrm{meV}$ and reach the band top at the $\Gamma$ point at $E \sim 4 \mathrm{meV}$ within the $K-\Gamma$ model. In Fig. 4(a) and (b), the continuous excitation spectra around the $\Gamma$ point drop to a much lower energy of $\sim 2 \mathrm{meV}$, therefore they should not be coherent spin waves but represent fractional excitations, supporting our polarization analyses.

For comparison, we also consider the widely proposed $K-J$ model for Kitaev materials [13, 15, 18, 19]:

$$
H_{K-J}=\sum_{<i j>}\left(J \boldsymbol{S}_{i} \cdot \boldsymbol{S}_{j}+K S_{i}^{\gamma} S_{j}^{\gamma}\right) .
$$

The calculated results with $K=9.8 \mathrm{meV}$ and $J=$ $-3.6 \mathrm{meV}$ using the cluster perturbation theory are shown in Fig. 4(c). Although the parameter set places the system in the correct zigzag order phase in the phase diagram constructed using the $K-J$ model $13,15,18,19$, and produces the spin-wave excitations dispersing from the $\mathrm{M}$ point, the calculation deviates from experimental observations in various aspects. First, the spin-wave excitations are gapless, in contrast to the gapped spectra shown in Fig. 4(a). Second, the low-energy excitations near the $\Gamma$ point are absent. Third, the Kitaev interaction is antiferromagnetic, contradicting previous reports on the ferromagnetic Kitaev interaction in $\alpha$ $\mathrm{RuCl}_{3}$ [31, 35, 37, 39. Therefore, the comparison between the experimental spectra and the theoretical results from different models not only confirms that the $K-\Gamma$ model is a more appropriate minimal spin model to describe $\alpha-\mathrm{RuCl}_{3}$ [31, 35, 37, but also represents as evidence that there present continuous fractional magnetic excitations near the $\Gamma$ point. We note, however, in addition to the dominant $K$ and $\Gamma$ terms, some other finite longer-range terms, although small, may be necessary to stabilize the zigzag order state [36, 66].

To conclude, we study the magnetic excitations by carrying out polarized INS measurements on high-quality $\alpha$ $\mathrm{RuCl}_{3}$ single crystals. We find that there exist pure continuous magnetic excitations near the $\Gamma$ point, which are robust against temperature, as opposed to the spin waves near the $\mathrm{M}$ point that vanish above $T_{\mathrm{N}}$. In addition, our calculation using the cluster perturbation theory shows that the $K-\Gamma$ model with a ferromegntic $K=-7.2 \mathrm{meV}$ and a comparable $\Gamma=5.6 \mathrm{meV}$ not only can reproduce the spin-wave excitations near the $\mathrm{M}$ point, but also the continuous excitations near the $\Gamma$ point. These results are evident that there exist exotic fractional excitations in $\alpha-\mathrm{RuCl}_{3}$ due to its proximity to the Kitaev QSL state.

However, considering the weak cross sections of the polarized experiment, further investigations with better statistics by using more single crystals, increasing the counting time, or optimizing the polarization options, should be helpful to solidify the conclusion. Potentially, one can learn details about the anisotropic interactions and magnetizations with more serious polarization analysis because the scattering intensities contain both an interference and a Chiral magnetic scattering term 68. In Ref. 66, it was proposed that the bond-dependent fractional excitations in $\alpha-\mathrm{RuCl}_{3}$ can be manifested in the equal-time structure factor $S^{\gamma}(\boldsymbol{Q})(\gamma=x, y, z$ represents different spin components) in the intermediate temperature range. We believe this is an interesting proposal worth of future efforts. Furthermore, for systems like $\alpha-\mathrm{RuCl}_{3}$, strongly spin-orbital-coupling may give rise to complex spin excitations like hybrid modes, the existence of frustrated anisotropic interactions will naturally lead to noticeable anharmonic effects [54. These may better explain the enhancement of the low-temperature excitations at the $\Gamma$ point as observed experimentally.

The work was supported by National Key Projects for Research and Development of China with Grant No. 2021YFA1400400, the National Natural Science Foundation of China with Grant Nos. 11822405, 12074174,12074175 , 11774152, 11904170, 12004249, 12004251 and 12004191, Natural Science Foundation of Jiangsu Province with Grant Nos. BK20180006, BK20190436 and BK20200738, Shanghai Sailing Program with Grant Nos. 20YF1430600 and 21YF1429200, Fundamental Research Funds for the Central Universities with Grant No. 020414380183, and the Office of International Cooperation and Exchanges of Nanjing University. The experiment at FLEXX was carried out under proposal No. 172-05993CR using beamtime allocated in the HZB-CIAE collaboration on the scientific use of instruments.

* These authors contributed equally to the work.

† slyu@nju.edu.cn

$\ddagger$ jxli@nju.edu.cn

$\S$ jwen@nju.edu.cn

[1] P.W. Anderson, "Resonating valence bonds: A new kind of insulator?" Mater. Res. Bull. 8, 153-160 (1973).

[2] P. W. Anderson, "The Resonating Valence Bond State in $\mathrm{La}_{2} \mathrm{CuO}_{4}$ and Superconductivity," Science 235, 11961198 (1987).

[3] Leon Balents, "Spin liquids in frustrated magnets," Nature 464, 199-208 (2010).

[4] Yi Zhou, Kazushi Kanoda, and Tai-Kai Ng, "Quantum spin liquid states," Rev. Mod. Phys. 89, 025003 (2017).

[5] Lucile Savary and Leon Balents, "Quantum spin liquids: a review," Rep. Pro. Phys. 80, 016502 (2017).

[6] Jinsheng Wen, Shun-Li Yu, Shiyan Li, Weiqiang Yu, and Jian-Xin Li, "Experimental identification of quantum spin liquids," npj Quant. Mater. 4, 12 (2019).

[7] C. Broholm, R. J. Cava, S. A. Kivelson, D. G. Nocera, M. R. Norman, and T. Senthil, "Quantum spin liquids," Science 367 (2020).

[8] Alexei Kitaev, "Anyons in an exactly solved model and beyond," Ann. Phys. 321, 2-111 (2006).

[9] Simon Trebst, "Kitaev materials," arXiv:1701.07056 
(2017).

[10] A. Yu. Kitaev, "Fault-tolerant quantum computation by anyons," Ann. Phys. 303, 2 - 30 (2003)

[11] Chetan Nayak, Steven H. Simon, Ady Stern, Michael Freedman, and Sankar Das Sarma, "Non-Abelian anyons and topological quantum computation," Rev. Mod. Phys. 80, 1083-1159 (2008).

[12] Maissam Barkeshli, Erez Berg, and Steven Kivelson, "Coherent transmutation of electrons into fractionalized anyons," Science 346, 722-725 (2014)

[13] G. Jackeli and G. Khaliullin, "Mott Insulators in the Strong Spin-Orbit Coupling Limit: From Heisenberg to a Quantum Compass and Kitaev Models," Phys. Rev. Lett. 102, 017205 (2009).

[14] Jeffrey G. Rau, Eric Kin-Ho Lee, and Hae-Young Kee, "Spin-orbit physics giving rise to novel phases in correlated systems: Iridates and related materials," Ann. Rev. Condens. Matter Phys. 7, 195-221 (2016).

[15] Jiří Chaloupka, George Jackeli, and Giniyat Khaliullin, "Kitaev-Heisenberg Model on a Honeycomb Lattice: Possible Exotic Phases in Iridium Oxides $A_{2} \mathrm{IrO}_{3}$," Phys. Rev. Lett. 105, 027204 (2010).

[16] Itamar Kimchi and Yi-Zhuang You, "Kitaev-Heisenberg$J_{2}-J_{3}$ model for the iridates $A_{2} \mathrm{IrO}_{3}$," Phys. Rev. B 84, 180407 (2011)

[17] Yogesh Singh, S. Manni, J. Reuther, T. Berlijn, R. Thomale, W. Ku, S. Trebst, and P. Gegenwart, "Relevance of the Heisenberg-Kitaev Model for the Honeycomb Lattice Iridates $A_{2} \mathrm{IrO}_{3}$," Phys. Rev. Lett. 108, 127203 (2012)

[18] Jiří Chaloupka, George Jackeli, and Giniyat Khaliullin, "Zigzag Magnetic Order in the Iridium Oxide $\mathrm{Na}_{2} \mathrm{IrO}_{3}$," Phys. Rev. Lett. 110, 097204 (2013).

[19] Jeffrey G. Rau, Eric Kin-Ho Lee, and Hae-Young Kee, "Generic Spin Model for the Honeycomb Iridates beyond the Kitaev Limit," Phys. Rev. Lett. 112, 077204 (2014)

[20] Yuriy Sizyuk, Craig Price, Peter Wölfle, and Natalia B. Perkins, "Importance of anisotropic exchange interactions in honeycomb iridates: Minimal model for zigzag antiferromagnetic order in $\mathrm{Na}_{2} \mathrm{IrO}_{3}$," Phys. Rev. B 90, 155126 (2014)

[21] Sae Hwan Chun, Jong-Woo Kim, Jungho Kim, H. Zheng, Constantinos C. Stoumpos, C. D. Malliakas, J. F. Mitchell, Kavita Mehlawat, Yogesh Singh, Y. Choi, T. Gog, A. Al-Zein, M. Moretti Sala, M. Krisch, J. Chaloupka, G. Jackeli, G. Khaliullin, and B. J. Kim, "Direct evidence for dominant bond-directional interactions in a honeycomb lattice iridate $\mathrm{Na}_{2} \mathrm{IrO}_{3}$," Nat. Phys. 11, 462-466 (2015).

[22] Stephen M Winter, Alexander A Tsirlin, Maria Daghofer, Jeroen van den Brink, Yogesh Singh, Philipp Gegenwart, and Roser Valentí, "Models and materials for generalized Kitaev magnetism," J. Phys. Conden. Matter 29, 493002 (2017)

[23] Stephen M. Winter, Ying Li, Harald O. Jeschke, and Roser Valentí, "Challenges in design of Kitaev materials: Magnetic interactions from competing energy scales," Phys. Rev. B 93, 214431 (2016)

[24] I. I. Mazin, Harald O. Jeschke, Kateryna Foyevtsova, Roser Valentí, and D. I. Khomskii, "Na2 $\mathrm{IrO}_{3}$ as a Molecular Orbital Crystal," Phys. Rev. Lett. 109, 197201 (2012)

[25] Kateryna Foyevtsova, Harald O. Jeschke, I. I. Mazin, D. I. Khomskii, and Roser Valentí, "ab initio analysis of the tight-binding parameters and magnetic interactions in $\mathrm{Na}_{2} \mathrm{IrO}_{3}$," Phys. Rev. B 88, 035107 (2013).

[26] K. W. Plumb, J. P. Clancy, L. J. Sandilands, V. Vijay Shankar, Y. F. Hu, K. S. Burch, Hae-Young Kee, and Young-June Kim, " $\alpha-\mathrm{RuCl}_{3}$ : A spin-orbit assisted Mott insulator on a honeycomb lattice," Phys. Rev. B 90, 041112 (2014),

[27] J. A. Sears, M. Songvilay, K. W. Plumb, J. P. Clancy, Y. Qiu, Y. Zhao, D. Parshall, and Young-June Kim, "Magnetic order in $\alpha-\mathrm{RuCl}_{3}$ : A honeycomb-lattice quantum magnet with strong spin-orbit coupling," Phys. Rev. B 91, 144420 (2015).

[28] R. D. Johnson, S. C. Williams, A. A. Haghighirad, J. Singleton, V. Zapf, P. Manuel, I. I. Mazin, Y. Li, H. O. Jeschke, R. Valentí, and R. Coldea, "Monoclinic crystal structure of $\alpha-\mathrm{RuCl}_{3}$ and the zigzag antiferromagnetic ground state," Phys. Rev. B 92, 235119 (2015)

[29] A. Banerjee, C. A. Bridges, J. Q. Yan, A. A. Aczel, L. Li, M. B. Stone, G. E. Granroth, M. D. Lumsden, Y. Yiu, J. Knolle, S. Bhattacharjee, D. L. Kovrizhin, R. Moessner, D. A. Tennant, D. G. Mandrus, and S. E. Nagler, "Proximate Kitaev quantum spin liquid behaviour in a honeycomb magnet," Nat. Mater. 15, 733-740 (2016).

[30] H. B. Cao, A. Banerjee, J.-Q. Yan, C. A. Bridges, M. D. Lumsden, D. G. Mandrus, D. A. Tennant, B. C. Chakoumakos, and S. E. Nagler, "Low-temperature crystal and magnetic structure of $\alpha-\mathrm{RuCl}_{3}$," Phys. Rev. B 93, 134423 (2016)

[31] Kejing Ran, Jinghui Wang, Wei Wang, Zhao-Yang Dong, Xiao Ren, Song Bao, Shichao Li, Zhen Ma, Yuan Gan, Youtian Zhang, J. T. Park, Guochu Deng, S. Danilkin, Shun-Li Yu, Jian-Xin Li, and Jinsheng Wen, "Spin-Wave Excitations Evidencing the Kitaev Interaction in Single Crystalline $\alpha-\mathrm{RuCl}_{3}$," Phys. Rev. Lett. 118, 107203 (2017)

[32] Heung-Sik Kim, Vijay Shankar V., Andrei Catuneanu, and Hae-Young Kee, "Kitaev magnetism in honeycomb $\mathrm{RuCl}_{3}$ with intermediate spin-orbit coupling," Phys. Rev. B 91, 241110 (2015)

[33] Luke J. Sandilands, Yao Tian, Anjan A. Reijnders, Heung-Sik Kim, K. W. Plumb, Young-June Kim, HaeYoung Kee, and Kenneth S. Burch, "Spin-orbit excitations and electronic structure of the putative Kitaev magnet $\alpha-\mathrm{RuCl}_{3}, "$ Phys. Rev. B 93, 075144 (2016).

[34] Heung-Sik Kim and Hae-Young Kee, "Crystal structure and magnetism in $\alpha-\mathrm{RuCl}_{3}$ : An ab initio study," Phys. Rev. B 93, 155143 (2016).

[35] Wei Wang, Zhao-Yang Dong, Shun-Li Yu, and Jian-Xin $\mathrm{Li}$, "Theoretical investigation of magnetic dynamics in $\alpha-\mathrm{RuCl}_{3}, "$ Phys. Rev. B 96, 115103 (2017)

[36] Lukas Janssen, Eric C. Andrade, and Matthias Vojta, "Magnetization processes of zigzag states on the honeycomb lattice: Identifying spin models for $\alpha-\mathrm{RuCl}_{3}$ and $\mathrm{Na}_{2} \mathrm{IrO}_{3}$," Phys. Rev. B 96, 064430 (2017).

[37] Arnab Banerjee, Jiaqiang Yan, Johannes Knolle, Craig A. Bridges, Matthew B. Stone, Mark D. Lumsden, David G. Mandrus, David A. Tennant, Roderich Moessner, and Stephen E. Nagler, "Neutron scattering in the proximate quantum spin liquid $\alpha-\mathrm{RuCl}_{3}$," Science 356, 1055-1059 (2017)

[38] Matthias Gohlke, Gideon Wachtel, Youhei Yamaji, Frank Pollmann, and Yong Baek Kim, "Quantum spin liquid signatures in Kitaev-like frustrated magnets," Phys. Rev. B 97, 075126 (2018) 
[39] Jennifer A. Sears, Li Ern Chern, Subin Kim, Pablo J. Bereciartua, Sonia Francoual, Yong Baek Kim, and Young-June Kim, "Ferromagnetic Kitaev interaction and the origin of large magnetic anisotropy in $\alpha-\mathrm{RuCl}_{3}$," Nat. Phys. 16, 837-840 (2020).

[40] Yumi Kubota, Hidekazu Tanaka, Toshio Ono, Yasuo Narumi, and Koichi Kindo, "Successive magnetic phase transitions in $\alpha-\mathrm{RuCl}_{3}$ : XY-like frustrated magnet on the honeycomb lattice," Phys. Rev. B 91, 094422 (2015).

[41] M. Majumder, M. Schmidt, H. Rosner, A. A. Tsirlin, H. Yasuoka, and M. Baenitz, "Anisotropic $\mathrm{Ru}^{3+} 4 d^{5}$ magnetism in the $\alpha-\mathrm{RuCl}_{3}$ honeycomb system: Susceptibility, specific heat, and zero-field NMR," Phys. Rev. B 91, 180401 (2015).

[42] J. A. Sears, Y. Zhao, Z. Xu, J. W. Lynn, and YoungJune Kim, "Phase diagram of $\alpha-\mathrm{RuCl}_{3}$ in an in-plane magnetic field," Phys. Rev. B 95, 180411 (2017)

[43] Takuya Aoyama, Yoshinao Hasegawa, Shojiro Kimura, Tsuyoshi Kimura, and Kenya Ohgushi, "Anisotropic magnetodielectric effect in the honeycomb-type magnet $\alpha-\mathrm{RuCl}_{3}, "$ Phys. Rev. B 95, 245104 (2017).

[44] Y. J. Yu, Y. Xu, K. J. Ran, J. M. Ni, Y. Y. Huang, J. H. Wang, J. S. Wen, and S. Y. Li, "Ultralow-Temperature Thermal Conductivity of the Kitaev Honeycomb Magnet $\alpha-\mathrm{RuCl}_{3}$ across the Field-Induced Phase Transition," Phys. Rev. Lett. 120, 067202 (2018).

[45] Arnab Banerjee, Paula Lampen-Kelley, Johannes Knolle, Christian Balz, Adam Anthony Aczel, Barry Winn, Yaohua Liu, Daniel Pajerowski, Jiaqiang Yan, Craig A. Bridges, Andrei T. Savici, Bryan C. Chakoumakos, Mark D. Lumsden, David Alan Tennant, Roderich Moessner, David G. Mandrus, and Stephen E. Nagler, "Excitations in the field-induced quantum spin liquid state of $\alpha-\mathrm{RuCl}_{3}$," npj Quant. Mater. 3, 8 (2018).

[46] Christian Balz, Paula Lampen-Kelley, Arnab Banerjee, Jiaqiang Yan, Zhilun Lu, Xinzhe Hu, Swapnil M. Yadav, Yasu Takano, Yaohua Liu, D. Alan Tennant, Mark D. Lumsden, David Mandrus, and Stephen E. Nagler, "Finite field regime for a quantum spin liquid in $\alpha-\mathrm{RuCl}_{3}$," Phys. Rev. B 100, 060405 (2019).

[47] Y. Cui, J. Zheng, K. Ran, Jinsheng Wen, Zheng-Xin Liu, B. Liu, Wenan Guo, and Weiqiang Yu, "High-pressure magnetization and NMR studies of $\alpha-\mathrm{RuCl}_{3}$," Phys. Rev. B 96, 205147 (2017).

[48] G. Bastien, G. Garbarino, R. Yadav, F. J. MartinezCasado, R. Beltrán Rodríguez, Q. Stahl, M. Kusch, S. P. Limandri, R. Ray, P. Lampen-Kelley, D. G. Mandrus, S. E. Nagler, M. Roslova, A. Isaeva, T. Doert, L. Hozoi, A. U. B. Wolter, B. Büchner, J. Geck, and J. van den Brink, "Pressure-induced dimerization and valence bond crystal formation in the Kitaev-Heisenberg magnet $\alpha-\mathrm{RuCl}_{3}, "$ Phys. Rev. B 97, 241108 (2018).

[49] Zhe Wang, Jing Guo, F. F. Tafti, Anthony Hegg, Sudeshna Sen, Vladimir A. Sidorov, Le Wang, Shu Cai, Wei Yi, Yazhou Zhou, Honghong Wang, Shan Zhang, Ke Yang, Aiguo Li, Xiaodong Li, Yanchun Li, Jing Liu, Youguo Shi, Wei Ku, Qi Wu, Robert J. Cava, and Liling Sun, "Pressure-induced melting of magnetic order and emergence of a new quantum state in $\alpha-\mathrm{RuCl}_{3}$," Phys. Rev. B 97, 245149 (2018).

[50] S.-H. Baek, S.-H. Do, K.-Y. Choi, Y. S. Kwon, A. U. B. Wolter, S. Nishimoto, Jeroen van den Brink, and B. Büchner, "Evidence for a Field-Induced Quantum Spin Liquid in $\alpha-\mathrm{RuCl}_{3}$," Phys. Rev. Lett. 119, 037201
(2017)

[51] Jiacheng Zheng, Kejing Ran, Tianrun Li, Jinghui Wang, Pengshuai Wang, Bin Liu, Zheng-Xin Liu, B. Normand, Jinsheng Wen, and Weiqiang Yu, "Gapless Spin Excitations in the Field-Induced Quantum Spin Liquid Phase of $\alpha-\mathrm{RuCl}_{3}$," Phys. Rev. Lett. 119, 227208 (2017)

[52] Zheng-Xin Liu and B. Normand, "Dirac and chiral quantum spin liquids on the honeycomb lattice in a magnetic field," Phys. Rev. Lett. 120, 187201 (2018)

[53] Y. Kasahara, T. Ohnishi, Y. Mizukami, O. Tanaka, Sixiao Ma, K. Sugii, N. Kurita, H. Tanaka, J. Nasu, Y. Motome, T. Shibauchi, and Y. Matsuda, "Majorana quantization and half-integer thermal quantum Hall effect in a Kitaev spin liquid," Nature 559, 227-231 (2018).

[54] Stephen M. Winter, Kira Riedl, Pavel A. Maksimov, Alexander L. Chernyshev, Andreas Honecker, and Roser Valentí, "Breakdown of magnons in a strongly spinorbital coupled magnet," Nat. Commun. 8, 1152 (2017).

[55] Luke J. Sandilands, Yao Tian, Kemp W. Plumb, Young-June Kim, and Kenneth S. Burch, "Scattering Continuum and Possible Fractionalized Excitations in $\alpha-\mathrm{RuCl}_{3}, "$ Phys. Rev. Lett. 114, 147201 (2015).

[56] J. Nasu, J. Knolle, D. L. Kovrizhin, Y. Motome, and R. Moessner, "Fermionic response from fractionalization in an insulating two-dimensional magnet," Nat. Phys. 12, 912-915 (2016).

[57] A. Little, Liang Wu, P. Lampen-Kelley, A. Banerjee, S. Patankar, D. Rees, C. A. Bridges, J.-Q. Yan, D. Mandrus, S. E. Nagler, and J. Orenstein, "Antiferromagnetic Resonance and Terahertz Continuum in $\alpha-\mathrm{RuCl}_{3}$," Phys. Rev. Lett. 119, 227201 (2017)

[58] Zhe Wang, S. Reschke, D. Hüvonen, S.-H. Do, K.-Y. Choi, M. Gensch, U. Nagel, T. Rõ om, and A. Loidl, "Magnetic Excitations and Continuum of a Possibly Field-Induced Quantum Spin Liquid in $\alpha-\mathrm{RuCl}_{3}$," Phys. Rev. Lett. 119, 227202 (2017)

[59] S. Reschke, F. Mayr, Zhe Wang, Seung-Hwan Do, K.-Y. Choi, and A. Loidl, "Electronic and phonon excitations in $\alpha-\mathrm{RuCl}_{3}, "$ Phys. Rev. B 96, 165120 (2017).

[60] Seung-Hwan Do, Sang-Youn Park, Junki Yoshitake, Joji Nasu, Yukitoshi Motome, Yong Seung Kwon, D. T. Adroja, D. J. Voneshen, Kyoo Kim, T. H. Jang, J. H. Park, Kwang-Yong Choi, and Sungdae Ji, "Majorana fermions in the Kitaev quantum spin system $\alpha-\mathrm{RuCl}_{3}$," Nat. Phys. 13, 1079 (2017).

[61] R. Nathans, C.G. Shull, G. Shirane, and A. Andresen, "The use of polarized neutrons in determining the magnetic scattering by iron and nickel," J. Phys. Chem. Solids 10, 138-146 (1959).

[62] R. M. Moon, T. Riste, and W. C. Koehler, "Polarization analysis of thermal-neutron scattering," Phys. Rev. 181, 920-931 (1969).

[63] A. Schneidewind and P. Čermák, "PANDA: Cold three axes spectrometer," J. Large Scale Res. Facil. 1, A12 (2015).

[64] O. Schaerpf and H. Capellmann, "Neutron scattering with a triple-axis spectrometer," Phys. Status Solidi A 135, 359 (1993).

[65] G. Shirane, S. M. Shapiro, and J. M. Tranquada, Neutron Scattering with a Triple-Axis Spectrometer: Basic Techniques (Cambridge University Press, Cambridge, 2002).

[66] Han Li, Dai-Wei Qu, Hao-Kai Zhang, Yi-Zhen Jia, ShouShu Gong, Yang Qi, and Wei Li, "Universal thermody- 
namics in the Kitaev fractional liquid," Phys. Rev. Research 2, 043015 (2020)

[67] Shun-Li Yu, Wei Wang, Zhao-Yang Dong, Zi-Jian Yao, and Jian-Xin Li, "Deconfinement of spinons in frustrated spin systems: Spectral perspective," Phys. Rev. B 98, 134410 (2018)

[68] Werner Schweika, "XYZ-polarisation analysis of diffuse magnetic neutron scattering from single crystals," Journal of Physics: Conference Series 211, 012026 (2010). 